\title{
A Influência de Mídias Multissensoriais na Aprendizagem de Crianças com Dislexia
}

\author{
Ellen Paixão Silva, Glauco Amorim, Joel dos Santos ${ }^{1}$ \\ ${ }^{1}$ Centro Federal de Educação Tecnológica Celso Suckow da Fonseca (CEFET-RJ) \\ (ellen.silva, gamorim, jsantos) deic.cefet-rj.br
}

\begin{abstract}
Studies published in the literature indicate that the use of multimedia content improves the reading performance of students with dyslexia. However, most multimedia applications use only two of the five human senses. MBook, a multisensory book developed for this work, uses an eye tracker to synchronize wind, smell, sounds, and light effects to the text being read. It was based on the hypothesis that a multisensory reading could reduce the overload in working memory, thus improving comprehension. Experimental results point to a gain in text comprehension and an increase in reading speed by the student with dyslexia when multisensory media were used. Also, the use of the tool pointed to an increase in reading motivation.
\end{abstract}

\section{Introdução}

Estudos em multimídia buscam aumentar a qualidade da experiência dos usuários em uma aplicação por meio da inclusão de efeitos sensoriais (e.g., hápticos, olfativos) de forma integrada ao conteúdo multimídia tradicional (i.e, áudio, vídeo, imagem e texto). No campo da educação, em geral com foco em aumentar o interesse de jovens pela leitura, pesquisas propõem incorporar conteúdo adicional a e-books, como áudio, imagens e $f e$ edback tátil. Essa abordagem é reforçada por pesquisas psicológicas que indicam que vários estímulos sensoriais têm um impacto aprimorado na memória e na aprendizagem [Shams and Seitz 2008, Heikkilä et al. 2015].

Dentre os transtornos caracterizados pela dificuldade de aprendizagem, a dislexia é o de maior incidência e estima-se que de 7 a $15 \%$ da população mundial seja acometida por esse transtorno [Rochelle and Talcott 2006]. Em indivíduos com dislexia, as dificuldades em interpretação de textos são frequentemente relacionadas com a sobrecarga da memória de trabalho [Habib 2000]. A leitura não automatizada, ou seja, lenta e silabada, demanda um esforço tão grande que o leitor não consegue reter e associar as informações do parágrafo lido, tendo com isso, dificuldades de compreensão. Nesse contexto, pesquisas como as de [Kast et al. 2007] e [Ekhsan et al. 2012] apontam uma melhora no desempenho de escolares com dislexia quando recursos multimídia são utilizados. Até onde sabemos, entretanto, nenhum estudo até o momento apresentou o uso de mídias multissensoriais como aromas e luzes sincronizados com o texto que está sendo lido.

\section{Objetivos}

A formação de memória engloba diferentes sentidos [Kast et al. 2007, Cahill et al. 2003] sendo apontada uma melhoria no aprendizado quando este é acompanhado de estímulos 
multissensoriais. Tais elementos promovem um contexto na formação da memória, facilitando sua recuperação. Com base nos resultados de [Kast et al. 2007, Ekhsan et al. 2012], espera-se que a incorporação de estímulos a outros sentidos além de visio-auditivos ajudem na formação da memória de indivíduos com dislexia, diminuindo a sobrecarga da memória de trabalho, possibilitando assim uma melhor compreensão do texto lido.

Dessa forma, pretende-se por meio de experimentos, avaliar o potencial do uso de livros multissensoriais para a diminuição da sobrecarga na memória de trabalho por indivíduos com dislexia. Assim, são esperadas uma melhora na compreensão e velocidade de leitura com a inclusão das mídias multissensoriais. Como objetivo secundário, este trabalho se propôs a avaliar o uso de livros multissensoriais como fator motivador para a leitura.

Para viabilizar a execução de tais experimentos, com base em trabalhos anteriores [Guedes 2018, Vieira et al. 2018], este trabalho apresentou o MBook, uma solução para a criação de livros multissensoriais. O MBook permite ao autor criar um livro multissensorial, desacoplando o conteúdo textual do livro da lógica da experiência multissensorial. A arquitetura desenvolvida para o MBook permite uma sincronização fina por incorporar um rastreador ocular. Além disso, a arquitetura dissocia o tipo de mídia multissensorial usada, permitindo que novos players de mídias diferentes sejam adicionados ao MBook, apenas definindo como se comunicar com eles. A Figura 1 apresenta a arquitetura do MBook.

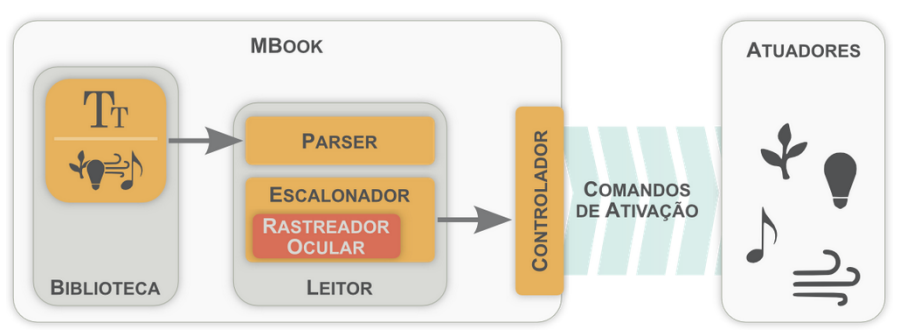

Figura 1. Arquitetura do aplicativo MBook.

\section{Contribuições e Resultados}

Para avaliar as hipóteses acima foram realizados dois estudos. Os estudos realizados contam com aprovação do Comitê de Ética em Pesquisa do Instituto de Neurologia Deolindo Couto da Universidade Federal do Rio de Janeiro (INDC - UFRJ), sob CAAE $n^{\circ}$ 33307520.7 .0000 .5261 .

O primeiro estudo consistiu na comparação de dois estudos de caso individuais com duas escolares do sexo feminino, do terceiro ano do ensino fundamental da rede particular de ensino, ambas com 9 anos idade, sendo uma delas com dislexia. Por simplicidade nos referiremos a criança com dislexia como $C C D$ e a criança não-disléxica como $C N D$ no restante do texto. O segundo estudo consistiu em um estudo de caso coletivo com cinco escolares de 8 a 11 anos de idade, entre a terceira e quarta série do ensino fundamental, da rede privada e pública de ensino, de ambos os sexos, sendo todos eles com dislexia. Por simplicidade nos referiremos a este grupo como $G C D$ no restante do texto. Em ambos os estudos foi utilizada a mesma história e foram utilizados os mesmos dispositivos seguindo a configuração apresentada na Figura 2. 


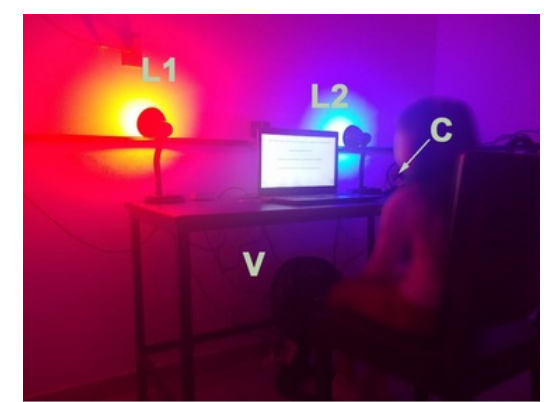

Figura 2. Montagem dos estudos com a utilização de computador para ler o texto, lâmpadas RGB, ventilador e difusor de cheiro.

Os experimentos foram desenhados de forma que cada participante fizesse três leituras do livro multissensorial, sendo a primeira leitura sem a presença de mídias multissensoriais e a segunda e terceira com a presença de mídias multissensoriais. O objetivo de realizar três leituras foi viabilizar a análise entre leituras com e sem mídias multissensoriais (primeira e segunda leituras) e a análise entre leituras com mídias multissensoriais (segunda e terceira leituras). A Figura 3 resume as etapas da condução dos experimentos.

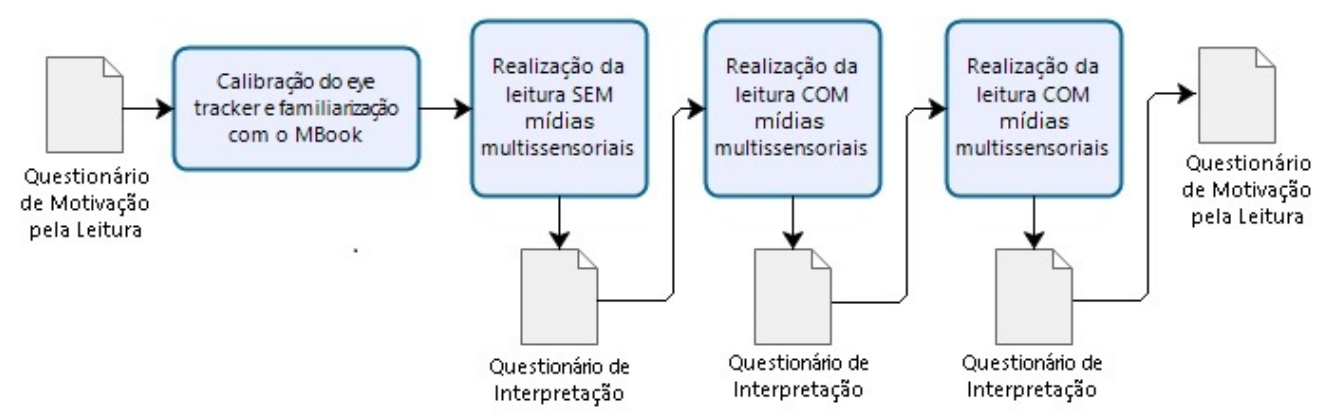

Figura 3. Esquema da condução do experimento.

Para a avaliação da motivação pela leitura, foi adaptado um questionário com 12 perguntas baseadas na escala para avaliação da motivação escolar infantojuvenil [Martinelli and Sisto 2011]. O questionário é constituindo de seis questões relacionadas à motivação intrínseca e seis questões à motivação extrínseca. Conforme apresentado na Figura 3, o questionário de avaliação da motivação pela leitura foi aplicado antes da primeira leitura e após a terceira leitura.

O questionário para avaliação da compreensão do texto é composto de cinco perguntas abertas com enfoque em pontos da história onde houve a ocorrência de mídias multissensoriais e busca avaliar o impacto do uso de mídias multissensoriais na compreensão. Conforme apresentado na Figura 3, o questionário de compreensão do texto foi aplicado após cada uma das três leituras.

Há também uma etapa de treinamento antes do início da primeira leitura que consiste em calibrar o eye-tracker e explorar o MBook para ambientação com a ferramenta.

\subsection{Discussão dos resultados}

Os resultados obtidos apontam para um ganho maior na compreensão do texto com a inserção das mídias multissensoriais. No primeiro estudo, tanto $C C D$ quanto $C N D$ apre- 
sentaram maior razão de ganho na compreensão do texto da primeira para a segunda leitura, quando foram inseridas as mídias multissensoriais, do que o observado da segunda para a terceira leitura, quando houve a repetição da leitura com mídias multissensoriais. Esse resultado é reforçado no segundo estudo, onde houve um ganho estatisticamente significativo obtido na compreensão do texto da primeira leitura para a segunda, não sendo observado o mesmo efeito da segunda leitura para a terceira leitura.

Tais resultados apontam que a inclusão de mídias multissensoriais facilitaram o processo de compreensão e que o efeito da repetição não foi causador desse aumento na compreensão. Os resultados também apontaram que o ganho de compreensão de $C C D$ foi maior que o de $C N D$ quando houve a inclusão das mídias multissensoriais, conseguindo a $C C D$ equiparar sua pontuação com a da $C N D$. Esses resultados são apoiados por pesquisas publicadas na literatura que indicam que a formação de memória engloba diferentes sentidos [Cahill et al. 2003] e que o uso de mídias multissensoriais melhoram o desempenho de escolares com dislexia [Ekhsan et al. 2012, Kast et al. 2007].

Em relação à velocidade de leitura, os resultados revelaram um aumento de velocidade quando da inserção de mídias multissensoriais. No primeiro estudo a CND apresentou um ganho de velocidade da primeira leitura para a segunda maior que o observado da segunda leitura para a terceira. Já para a $C C D$ houve a mesma razão de ganho de velocidade da primeira leitura para a segunda e da segunda para a terceira. O segundo estudo reforça a percepção acima, revelando um aumento estatisticamente significativo na velocidade da primeira leitura para a segunda, fato não observado da segunda leitura para a terceira. Tais resultados apontam que a inclusão de mídias multissensoriais facilitaram o processo de leitura e que o efeito da repetição não foi causador desse aumento na velocidade.

De acordo com [dos Santos 2019], as medidas de fluência (palavras lidas por minuto - ppm) em leitura nos escolares do $2^{\circ}$ ao $5^{\circ}$ ano do Ensino Fundamental é de 70, 6ppm para os escolares do $2^{\circ}$ ano. Para o $3^{\circ}$ ano é de $84,9 \mathrm{ppm}$, para o $4^{\circ}$ ano é de $116,5 \mathrm{ppm}$ e para o $5^{\circ}$ ano é de $137,0 \mathrm{ppm}$. A fluência da primeira leitura de $C C D$ foi de $64,33 \mathrm{ppm}$, passando para 74, 33ppm na segunda leitura e terminando em $83,91 \mathrm{ppm}$ na terceira leitura, chegando bem próximo da média de fluência indicada por [dos Santos 2019] para escolares do $3^{\circ}$ ano.

De acordo com [Mousinho et al. 2009], a precisão e velocidade de leitura, apesar de não serem suficientes para garantir a interpretação desejada de textos, favorecem muito a possibilidade de compreensão. Com isso podemos relacionar a melhora obtida na interpretação do texto ao aumento da velocidade de leitura. Os ganhos de velocidade e compreensão estatisticamente significativos quando adicionadas mídias multissensoriais (diferentemente da repetição da leitura), indicam que as mídias multissensoriais promoveram um contexto na formação da memória, facilitando sua recuperação, diminuindo a sobrecarga na memória de trabalho necessária para a leitura e, auxiliando assim, o indivíduo com dislexia a ganhar automatismo e melhorar sua compreensão do texto.

Considerando os dados relativos a motivação pela leitura, os resultados obtidos nos dois estudos indicam que o uso do livro multissensorial atuou como fator motivador para a leitura. Em ambos os estudos houve um aumento na motivação intrínseca, sendo esse ganho estatisticamente significativo no segundo estudo. Esse aumento da motivação 
intrínseca pode ter se dado pela perspectiva e pela curiosidade de quais efeitos poderiam surgir durante a leitura multissensorial. A motivação extrínseca e a motivação geral, que é a soma das motivações intrínseca e extrínseca, não obtiveram aumento significativo. De acordo com os achados de [Wang and Guthrie 2004, Meece and Miller 1999], estas variações de motivação podem ter contribuído para o ganho de compreensão. Tais estudos sugerem que, embora a motivação extrínseca esteja associada positivamente com notas de avaliação da leitura, é menos provável que influencie significativamente na compreensão do texto, como ocorre em escolares com predominância de motivação intrínseca.

\section{Conclusão}

Os resultados de ambos os estudos apontam a viabilidade do uso do MBook como uma ferramenta terapêutica importante para o tratamento de escolares com dislexia. Foi observado um ganho maior na compreensão do texto com a inserção das mídias multissensoriais do que o observado pela simples repetição da leitura. Em conjunto com o ganho na compreensão, foi observado que a inclusão de mídias multissensoriais também influenciaram na velocidade de leitura. Tais resultados são suportados por estudos publicados na literatura que indicam que melhorias na compreensão e aumentos na velocidade de leitura estão relacionados. Com isso podemos relacionar a melhora obtida na interpretação do texto ao aumento da velocidade de leitura. Ambos os ganhos indicam que as mídias multissensoriais promoveram um contexto na formação da memória, facilitando sua recuperação, diminuindo a sobrecarga na memória de trabalho necessária para a leitura. Assim, auxiliando o indivíduo com dislexia a ganhar automatismo e melhorar sua compreensão do texto. Os resultados obtidos apontam ainda que o uso de mídias multissensoriais associadas à leitura é um importante fator para aumento da motivação intrínseca de leitura.

As contribuições desse trabalho foram publicadas em:

- Silva, E. P., Amorim, G. F., dos Santos, J. (2019) Adding temporal semantic to textual media objects with eye tracking technology. WebMedia. pages 217-220. https://doi.org/10.1145/3323503.3360636

- Silva, E. P., Amorim, G. F., Guedes, G., dos Santos, J., Mousinho, R. (2021) A influência de mídias multissensoriais na aprendizagem de crianças com transtorno de leitura. Rev. Psicopedagogia, 38(115):104-120. https://doi .org/10 . 51207/2179-4057.20210009

- Silva, E. P., Vieira, N., Amorim, G. F., Mousinho, R., Guedes, G., Ghinea, G., dos Santos, J.. (2021) Using Multisensory Content to Impact the Quality of Experience of Reading Digital Books. To appear in ACM Trans. Multimedia Comput. Commun. https://doi.org/10.1145/3458676

\section{Referências}

Cahill, L., Gorski, L., and Le, K. (2003). Enhanced human memory consolidation with post-learning stress: Interaction with the degree of arousal at encoding. Learning \& memory, 10(4):270-274.

dos Santos, L. F. (2019). Análise da evolução de fluência de leitura em alunos do $2^{\circ}$ ao $9^{o}$ ano do Ensino Fundamental. Master's thesis, Faculdade de Medicina da Universidade Federal de Minas Gerais. 
Ekhsan, H. M., Ahmad, S. Z., Halim, S. A., Hamid, J. N., and Mansor, N. H. (2012). The implementation of interactive multimedia in early screening of dyslexia. In ICIMTR, pages 566-569.

Guedes, G. P. (2018). Multisensorial books: improving readers' quality of experience. In 2018 XIII Latin American Conference on Learning Objects and Technology (LACLO).

Habib, M. (2000). The neurological basis of developmental dyslexia: an overview and working hypothesis. Brain, 123(12):2373-2399.

Heikkilä, J., Alho, K., Hyvönen, H., and Tiippana, K. (2015). Audiovisual semantic congruency during encoding enhances memory performance. Experimental Psychology, 62(2):123-130.

Kast, M., Meyer, M., Vögeli, C., Gross, M., and Jäncke, L. (2007). Computer-based multisensory learning in children with developmental dyslexia. Restorative Neurology and Neuroscience, 25(3-4):355-369.

Martinelli, S. and Sisto, F. (2011). Escala para avaliação da motivação escolar (EAME-IJ). São Paulo: Casa do Psicólogo (no prelo).

Meece, J. L. and Miller, S. D. (1999). Changes in elementary school children's achievement goals for reading and writing: Results of a longitudinal and an intervention study. Scientific Studies of Reading, 3(3):207-229.

Mousinho, R., Mesquita, F., Leal, J., Pinheiro, L., et al. (2009). Compreensão, velocidade, fluência e precisão de leitura no segundo ano do ensino fundamental.

Rochelle, K. S. and Talcott, J. B. (2006). Impaired balance in developmental dyslexia? A meta-analysis of the contending evidence. Journal of Child Psychology and Psychiatry, 47(11):1159-1166.

Shams, L. and Seitz, A. (2008). Benefits of multisensory learning. Trends in Cognitive Sciences, 12(11):411-417.

Vieira, N., Pinto, A., Silva, F., Okuno, H., Amorim, I., Ramos, T., Haddad, D., Amorim, G., Guedes, G. P., and dos Santos, J. (2018). Evaluating the influence of mulsemedia content in reading. In WebMedia, pages 133-136. ACM.

Wang, J. H. and Guthrie, J. T. (2004). Modeling the effects of intrinsic motivation, extrinsic motivation, amount of reading, and past reading achievement on text comprehension between US and Chinese students. Reading research quarterly, 39(2):162-186. 